\title{
SINGLE ITEM INVENTORY MODELS \\ A TIME- AND EVENT- AVERAGES APPROACH
}

\author{
EMŐKE BÁZSA* AND PETER DEN ISEGER ${ }^{\dagger}$ \\ Econometric Institute Report EI 2002-46
}

\begin{abstract}
This paper extends a fundamental result about single-item inventory systems. This approach allows more general performance measures, demand processes and order policies, and leads to easier analysis and implementation, than prior research. We obtain closed form expressions for the Laplace transforms of the expressions of the performance measures, and with the help of an efficient inversion algorithm, the approximations of these cost and service measures are almost up to machine precision.
\end{abstract}

\section{INTRODUCTION}

Consider a single item inventory model which allows for backorders. Let us start from the basic flow-conservation relation, often used in inventory theory, relating the netstock process IN (physical stock - backorders), the inventory position process IP (netstock + items on order), and the demand process $\mathbf{D}$,

$$
\mathbf{I N}(t+L)=\mathbf{I P}(t)-\mathbf{D}(t, t+L)
$$

where $L>0$ denotes the leadtime. It was shown for certain demand processes and order policies that the limiting variables exist, and

$$
\mathbf{I N}_{\infty}=\mathbf{I} \mathbf{P}_{\infty}-\mathbf{D}_{\infty}(L)
$$

Here, the random variables $\mathbf{I} \mathbf{N}_{\infty}, \mathbf{I} \mathbf{P}_{\infty}$ and $\mathbf{D}_{\infty}(L)$ are distributed with the pointwise limiting distributions of the corresponding processes. Relation (1.1) in its own wouldn't be a

Date: December 18, 2002.

*Erasmus University Rotterdam, Econometric Institute, P.O. Box 1738, 3000 DR Rotterdam, The Netherlands, e-mail: bazsa@few.eur.nl.

${ }^{\dagger}$ Erasmus University Rotterdam, Econometric Institute, P.O. Box 1738, 3000 DR Rotterdam, The Netherlands, e-mail: iseger@few.eur.nl. 
fundamental result, but the following two properties turn it into a strong statement:

$$
\mathbf{I P}(t) \text { and } \mathbf{D}(t, t+L) \text { are asymptotically independent, }
$$

$\mathbf{t}_{n}, n \in \mathbb{N} \cup\{0\}$ representing the arrival epochs of customers.

The statements (1.1) - (1.3) were first proven back in 1979 by Sahin, for the case of a compound renewal demand process under an $(s, S)$ policy (cf. Sahin(1979)). Later, in 1986, Zipkin extended Sahin's result for the case of demand generated by a compound-counting process, with i.i.d. individual demands, independent of the demand epochs. The ordering policy he considers is based only on the inventory position. Further he assumes that the distribution of the stochastic counting process $\mathbf{N}(t)$, associated with the arrival process of the customers, converges pointwise to a limiting distribution. See our main reference, Zipkin(1986), for earlier work.

Our work proves that statements (1.1) - (1.3) are valid in case of more general demand processes or policies. Our extension of the already known results is easiest explained intuitively, through the difference in the assumptions made by Zipkin(1986) and those in the present paper. That is, instead of requiring that the pointwise limiting distribution for IP and $\mathbf{N}$ would exist, we assume the following: the limiting distribution in the Cesaro sense of the joint process $(\mathbf{I P}, \mathbf{N})$ exists. Since the Cesaro sense limiting distribution is a long run average, it is clearly less restrictive than the pointwise limit assumption. Furthermore, since we also prove asymptotical independence of the processes $\mathbf{I P}$ and $\mathbf{D}$, or equivalently, $\mathbf{I P}$ and $\mathbf{N}$, in order to be able to 'take apart' the joint limiting distribution in the Cesaro sense of IP and $\mathbf{N}$, we will consider the following cases: (i) the limiting distribution in the Cesaro sense of $\mathbf{N}(t)$ AND the pointwise limiting distribution of $\mathbf{I P}(t)$ exist, or (ii) the pointwise limiting distribution of $\mathbf{N}(t)$ AND the limiting distribution in the Cesaro sense of $\mathbf{I P}(t)$ exists. Under case (i) we can list models such as demand modeled by a nonhomogeneous compound Poisson process, i.e., the pointwise limit for the corresponding counting process $\mathbf{N}$ does not exist, while the Cesaro limit does. Under assumption (ii) models such as an $(s, S)$ policy with unit demand can be analyzed: the pointwise limit of the IP process does not exist while the limiting distribution in the Cesaro sense does. The importance of this result (asymptotical independence) is further illustrated by the example of a two level distribution system. 
Section 4 brings the other main result of this paper: the cost structure associated with this general model is also innovative, in the sense that it provides a unified treatment of average costs and service measures, by exploiting the asymptotical independence result of the previous section. We obtain the most important performance measures (that is, average costs and service measures) by observing the behaviour of the net inventory process. The end result is a general cost expression which yields any desired cost or service measure by solely substituting the proper cost-rate function. Furthermore, the convolution structure of these performance measure expressions, enables us to obtain a closed form expression for their Laplace transformations, without a significant effort. We then make use of a recently developed Laplace transform inversion technique (Den Iseger(2000)), which facilitates us to invert these Laplace transforms in any point. The obtained results are exact almost up to machine precision.

All the results of the present paper hold true in the case of stochastic leadtimes, provided that orders do not cross in time, as it is explained in detail in Section 5.1. Moreover, Section 5.2 deduces a surprising relationship between fixed-leadtime-models with time- nonhomogeneous compound Poisson demand, and stochastic-leadtime-models with a compound point process demand of Zipkin(1986). Section 6 provides numerical examples.

\section{Notation AND MAin TOOLS}

This section presents the three most important tools for this paper. Let us begin with the stochastic processes describing the inventory systems of our interest, that is single item inventory systems with backlogging. The demand process $\mathbf{D}$, is a general, stochastic compound point process, where $\mathbf{D}(t)$ represents the aggregate demand up to time $t$

$$
\mathbf{D}(t):=\sum_{n=1}^{\mathbf{N}(t)} \mathbf{Y}_{n}
$$

The individual demands $\mathbf{Y}_{n}, n \in \mathbb{N}$ are independent and identically distributed random variables, and independent of the arrival process of customers, N. Customers' interarrival times are described by the process $\mathbf{X}_{n}, n \in \mathbb{N}$, are not necessarily independent or identically distributed! The arrival times of the customers are thus given by $\mathbf{t}_{n}:=\mathbf{X}_{1}+\ldots+\mathbf{X}_{n}, n \in \mathbb{N}$ 
and the related stochastic counting process $\{\mathbf{N}(t): t \geq 0\}$ is given by

$$
\mathbf{N}(t):=\sum_{n=1}^{\infty} 1_{\left\{\mathbf{t}_{n} \leq t\right\}} .
$$

The netstock or net inventory process $\mathbf{I N}:=\{\mathbf{I N}(t): t \geq 0\}$, is defined as the stock on hand minus the backordered amount at time $t$, and the inventory position process $\mathbf{I P}:=\{\mathbf{I P}(t)$ : $t \geq 0\}$, is the net stock plus outstanding orders at time $t$. The control rule associated with the system is such that it only depends on the inventory position. An extension of this model, where the control rule is not based on the inventory position, can be found in Bázsa and Den Iseger(2001). In our analysis $L$ can be fixed or stochastic and we refer to it as the leadtime. We also need to make the common 'no order crossing' assumption: all and only those orders placed by time $t$ will arrive by time $t+L$. This assumption is essential for the validity of the so called flow conservation law expression, which is a key tool for this paper. If the stochastic demand process $\mathbf{D}$ is càdlàg (that is right continuous with left limits) then

$$
\phi_{L} \mathbf{I N}(t)=\mathbf{I P}(t)-\phi_{t} \mathbf{D}(0, L], \mathbb{P} \text { - almost surely }
$$

for every $t \geq 0$ where $\phi_{s}, s \geq 0$ is a shift operator such that $\phi_{s}(\mathbf{X})(t):=\mathbf{X}(t+s)$ for every $t \geq 0$ and $\mathbf{X}$ a stochastic process. For the same general stochastic process $\mathbf{X}$ the notation $\mathbf{X}(a, b]$ means $\mathbf{X}(b)-\mathbf{X}(a)$.

One of the main goals of this paper is to show that statements (1.1) - (1.3) and their application to performance measures hold under more general circumstances than in Zipkin(1986). That is, for the stochastic counting process $\mathbf{N}(t)$ or the inventory position process IP, instead of the existence of a pointwise limiting distribution we only assume the existence of the time or event stationary distributions. These distributions are defined as follows (see Sigman(1995)).

Definition 2.1. Consider a compound point process $\mathbf{X}=\{\mathbf{X}(t): t \geq 0\}$ and the sequence of events $\left\{\mathbf{s}_{n}, n \in \mathbb{N}\right\}$ related to $\mathbf{X}$. The distribution

$$
F_{\infty}^{c}(x)=\lim _{t \uparrow \infty} \frac{1}{t} \int_{0}^{t} F_{\mathrm{X}(\mathbf{s})}(x) d s
$$

is called the time stationary distribution for $\mathbf{X}$ (see Sigman(1995)). The distribution given by

$$
F_{\infty}^{e}(x)=\lim _{n \uparrow \infty} \frac{1}{n} \sum_{k=1}^{n} F_{\mathrm{X}\left(\mathbf{s}_{\mathrm{k}}\right)}(x)
$$


is defined as the event stationary distribution for X (see Sigman(1995)).

Since the the time and event stationary distributions are defined by Cesaro limits (time and resp. event averages!), their existence is a much less restrictive condition than that of a pointwise limiting distribution. This is the reason for referring to these distributions suggestively, under a common noun, as limiting distributions in Cesaro sense. A good example for the generality of these distributions is the time-nonhomogeneous compound Poisson process: its pointwise limit in distribution does not exist, while both the time and event stationary distributions exist.

The technical tool we use for the computation of the cost expressions is the LaplaceStieltjes transform and its inversion. ${ }^{1}$ The otherwise cumbersome convolution structure of the cost expressions becomes easily tractable through Laplace transformations. These expressions, involving Laplace transforms, can then be inverted with an efficient algorithm (see Den Iseger(2000)) and we obtain piece-wise polynomial approximations in fractions of time. The calculations are numerically stable, while the approximation is precise almost up to machine precision.

\section{The inVEntory position PRocess}

The definitions of this general control system imply for the inventory position process in the epochs of customers' arrival that $\mathbf{I P}\left(\mathbf{t}_{n}\right)$ only depends on the previous state $\operatorname{IP}\left(\mathbf{t}_{n-1}\right)$, the individual demand of the $n$th customer $\mathbf{Y}_{n}$, and the magnitude of the replenishment order $\mathbf{Z}_{n}$, if there was any order placed at $\mathbf{t}_{n}$. Since the individual demands $\mathbf{Y}_{n}$ are independent and identically distributed, and $\mathbf{Z}_{n}$ only depends on $\mathbf{I P}\left(\mathbf{t}_{n-1}\right)$ and $\mathbf{Y}_{n},\left\{\mathbf{I P}\left(\mathbf{t}_{n}\right): n \in \mathbb{N}\right\}$ is a Markov chain. If the chain $\left\{\mathbf{I P}\left(\mathbf{t}_{n}\right): n \in \mathbb{N} \cup\{0\}\right\}$ has a unique limiting distribution (see $\operatorname{Ross}(1970))$, then it is given by

$$
\pi:=\lim _{n \uparrow \infty} \mathbb{P}\left\{\mathbf{I P}\left(\mathbf{t}_{n}\right) \leq x\right\}=\mathbb{P}\left\{\mathbf{I P}_{\infty} \leq x\right\}
$$

where $\mathbf{I P} \mathbf{P}_{\infty}$ is a random variable distributed with the limiting distribution of the Markov chain $\left\{\mathbf{I P}\left(\mathbf{t}_{n}\right), n \in \mathbb{N}\right\}$. Otherwise, if only the limiting distribution in the Cesaro sense exists, that

\footnotetext{
${ }^{1}$ All the arguments and results remain valid in case of Fourier transforms.
} 
is, the event stationary distribution for $\mathbf{I P}_{n}$, then it is denoted by

$$
\pi^{c}:=\lim _{n \uparrow \infty} \frac{1}{n} \sum_{k=1}^{n} \mathbb{P}\left\{\mathbf{I P}\left(\mathbf{t}_{k}\right) \leq x\right\}=\mathbb{P}\left\{\mathbf{I P}_{\infty}^{c} \leq x\right\} .
$$

The step function structure of the sample paths of the inventory position implies that

$$
\mathbf{I P}(t)=\mathbf{I P}\left(\mathbf{t}_{\mathbf{N}(\mathrm{t})}\right), \text { for all } t \geq 0 .
$$

In order to prove that $\mathbf{I P}(t)$ is asymptotically independent of $\mathbf{N}(t)$, thus also independent of $\mathbf{D}(t)$, we need the definition of asymptotical independence understood in the Cesaro sense, that is:

Definition 3.1. If $\mathbf{X}$ and $\mathbf{Y}$ are two stochastic processes such that $\mathbf{X}$ has a pointwise limiting distribution and the time- stationary distribution for $\mathbf{Y}$ exists, then they are asymptotically independent if and only if

$$
\lim _{T \uparrow \infty} \frac{1}{T} \int_{0}^{T} \mathbb{P}\{\mathbf{X}(t) \leq x, \mathbf{Y}(t) \leq y\} d t=\mathbb{P}\left\{\mathbf{X}_{\infty} \leq x\right\} \mathbb{P}\left\{\mathbf{Y}_{\infty}^{c} \leq y\right\}
$$

where $\mathbf{X}_{\infty}$ is a random variable distributed with the pointwise limiting distribution of $\mathbf{X}$, and $\mathbf{Y}_{\infty}^{c}$ is a random variable distributed with the time- stationary distribution of $\mathbf{Y}$.

Observe that this definition can be easily adjusted in case of discrete stochastic processes and an event- stationary distribution. The asymptotical independence is justified by the following theorem, for any inventory system where the control rule solely depends on the inventory position:

Theorem 3.2. Assuming that the Markov chain $\left\{\mathbf{I P}\left(\mathbf{t}_{n}\right): n \in \mathbb{N}\right\}$ is ergodic, and the timestationary distribution for the stochastic counting process $\mathbf{N}$ exists, while $\mathbf{N}(t) \longrightarrow \infty$ a.s. as $t \longrightarrow \infty$, the inventory position process $\mathbf{I P}(t)$ and the leadtime demand $\mathbf{D}(t, t+L]$ are asymptotically independent. Moreover,

$$
\lim _{t \uparrow \infty} \mathbb{P}\{\mathbf{I P}(t) \leq x\}=\mathbb{P}\left\{\mathbf{I P}_{\infty} \leq x\right\}=\pi,
$$

for all $x \in \mathbb{R}$, where $\mathbf{I P} \mathbf{\infty}_{\infty}$ and $\pi$ were defined by relation (3.1). Conversely, if the eventstationary distribution $\pi^{c}$ for $\left\{\mathbf{I P}_{n}\right\}$ exists (defined by relation (3.2)), together with a pointwise limiting distribution of $\mathbf{N}$, with $\mathbf{N}(t) \longrightarrow \infty$ a.s. as $t \longrightarrow \infty$, the inventory position process $\mathbf{I P}(t)$ and the leadtime demand $\mathbf{D}(t, t+L]$ are asymptotically independent. 
For the proof see the Appendix.

Remark 3.3. In the discrete case the conclusions of the theorem remain valid, obviously assuming that the event- stationary distribution for the stochastic counting process $\mathbf{N}$ exists.

We will now give examples which are related to two of the most well-known policies in the literature.

3.1. The $(s, S)$ policy. This control policy only depends on the inventory position process. Hence, as derived at the beginning of section 3 the inventory position in the moments of customer arrivals $\left\{\mathbf{I P}\left(\mathbf{t}_{n}\right), n \in \mathbb{N}\right\}$ is a Markov chain which possesses a unique limiting distribution. This distribution, though in a different way, was also derived by Sahin (see Sahin(1990)). For notational convenience, define the sequence of random variables $\left\{\mathbf{V}_{n}: n \in\right.$ $\mathbb{I N}\}$ as the difference between the order-up-to level $S$ and the inventory position at moment $\mathbf{t}_{n}, n \in \mathbb{N}$ :

$$
\mathbf{V}_{n}:=S-\mathbf{I P}\left(\mathbf{t}_{n}\right), n \in \mathbb{N}
$$

Since $\left\{\mathbf{I P}\left(\mathbf{t}_{n}\right): n \in \mathbb{N}\right\}$ is a Markov chain, obviously $\left\{\mathbf{V}_{n}: n \in \mathbb{N}\right\}$ is also a Markov chain equipped with a unique limiting distribution. By the definition of the policy it immediately follows that

$$
\mathbf{V}_{n+1}=\left(\mathbf{V}_{n}+\mathbf{Y}_{n+1}\right) \mathbf{1}_{\left\{\mathbf{V}_{n}+\mathbf{Y}_{n+1} \leq S-s\right\}}, \quad n \in \mathbb{N}
$$

We aim to show now that the unique limiting distribution of the Markov chain $\left\{\mathbf{V}_{n}: n \in \mathbb{N}\right\}$ is of the form

$$
\lim _{n \uparrow \infty} \mathbb{P}\left\{\mathbf{V}_{n} \leq x\right\}=\mathbb{P}\left\{\mathbf{V}_{\infty} \leq x\right\}=\frac{U_{0}(x)}{U_{0}(S-s)},
$$

where $U_{0}$ denotes the renewal function related to the renewal sequence $\left\{\mathbf{Y}_{0}, \mathbf{Y}_{0}+\mathbf{Y}_{1}, \ldots\right\}$ given by

$$
U_{0}(x):=\sum_{k=0}^{\infty} F_{Y}^{k \star}(x) .
$$

Relation (3.8) is exploited in Bázsa and Den Iseger (2001a) for the purpose of a very efficient optimization algorithm. Relation (3.7) implies straightforwardly that for every $0 \leq x \leq S-s$

$$
F_{V}(x)=C+\left(F_{V} \star F_{Y}\right)(x),
$$


where $C:=1-\left(F_{V} \star F_{Y}\right)(S-s)$ is a normalization constant. Since relation (3.9) is a renewal type equation, it follows (see Ross(1970)) that its uniquely determined solution is given by

$$
F_{V}(x)=C U_{0}(x)
$$

The constant $C$ can be easily determined by the condition $F_{V}(S-s)=1$, therefore we obtain that the unique invariant distribution of the Markov chain $\mathbf{V}_{n}$ is given by relation (3.8). As a standard result from renewal theory (see $\operatorname{Ross}(1970)$ ), if $x$ is big enough, that is, $S-s$ is large, then the renewal function $U(x) / x \longrightarrow 1 / \mathbb{E} \mathbf{X}_{1}$. This implies that (3.8) converges to $x /(S-s)$, that is, the limiting distribution converges to a uniform distribution, and it $\mathrm{s}$ independent of the demand process!

In the next subsection it is proved that the limiting distribution of the Markovian inventory position process related to an $(s, Q)$ model is given by the uniform distribution. This result suggests that for large $Q$ and $S-s$ these models are very similar.

3.2. The $(s, n Q)$ policy. Hadley and Whitin(1963) proved that in case of an $(s, n Q)$ policy the transition matrix of the Markov chain $\left\{\mathbf{I P}\left(\mathbf{t}_{n}\right): n \in \mathbb{N}\right\}$ is double stochastic, hence it follows straightforwardly that its limiting distribution is given by the uniform distribution on $(s, s+Q]$, that is

$$
\lim _{n \uparrow \infty} \mathbf{I P}\left(\mathbf{t}_{n}\right)=s+Q \mathbf{U}, \quad n \in \mathbb{N},
$$

with $\mathbf{U}$ a uniformly distributed random variable on $(0,1]$. Together with the average holding and ordering cost expressions this result was also found by Chen and Zheng(1992), for a compound Poisson demand process. It is possible though to generalize this case even further. Assume that the inventory position $\mathbf{I} \mathbf{P}_{n}$ has the steady state distribution $s+Q \mathbf{U}_{n}$, with $\mathbf{U}_{n}$ uniformly distributed as before, and assume that the individual demand $Y_{i}$ are not identically distributed anymore, perhaps not even independent. It follows now that $\mathbf{I P}_{n+1} \stackrel{d}{=}\left[Q \mathbf{U}_{n}+\right.$ $\left.\mathbf{Y}_{n+1}\right] \bmod Q \stackrel{d}{=} Q \mathbf{U}_{n+1}$, and $\mathbf{I} \mathbf{P}_{n+2} \stackrel{d}{=} Q \mathbf{U}_{n+2}$, hence the distribution of the inventory position process remains uniformly distributed on $(s, s+Q]$. Furthermore, $\mathbf{I P}_{n}$ is independent of $\left\{\mathbf{Y}_{k}: k=1, \ldots, n\right\}$. 


\section{Performance measures}

4.1. The cost structure. Now we consider a general approach to performance measures. In general, the cost of an inventory control system (most commonly: long run average cost) is associated with the net inventory process. It is well-known that the sample paths of the net inventory process is a step function, with two types of jumps: (downwards) jumps occurring due to the arrival of customers, called type I jumps, and (upwards) jumps caused by the arrival of a replenishment order, called type II jumps. We associate three kinds of costs with the netstock process. The first type of cost is incurred between events, that is, between jumps. The second and third kinds of costs are associated with the type I and type II jumps, respectively, as follows:

first: When $\mathbf{I N}(t)=\mathbf{I N}\left(\mathbf{J}_{n}\right)=x$ a.s. for $\mathbf{J}_{n} \leq t<\mathbf{J}_{n+1}$, where $x \in \mathbb{R}$ is a constant and $\mathbf{J}_{n}, n \in \mathbb{N}$ are the points of time when a jump occurs, then it is natural and trivial to introduce a cost-rate function $f(x)$ related to this event. This cost will give us a very important characteristic, the average holding cost (and penalty cost), therefore we refer to this type of cost in the remainder of the paper as the average holding cost.

second: Similarly, we introduce a cost-rate function $g_{1}$ related to the type I jumps of the sample paths of the netstock process, that is, the cost of the jump in time point $\mathbf{t}_{n}$ is given by $g_{1}\left(\mathbf{I N}\left(\mathbf{t}_{n}^{-}\right), \mathbf{Y}_{n}\right)$. This type of "cost" usually provides us with service measures, since it is related to the arrival of customers. Therefore we refer to the cost of the type I jumps as service measures. Observe, that by altering the cost-rate function $g_{1}$, we obtain any specific service measure one needs. Later we also show that this cost-rate function is most of the time given by a simple algebraic expression.

third: Introduce also a function $G_{2}$, related to the type II jumps, that is, the cost of the control policy: for a replenishment order placed at time point $\mathbf{t}_{n}$ it is given by $G_{2}\left(\mathbf{Z}_{n}\right)$. By the definition of $\mathbf{Z}_{n}, \mathbf{Z}_{n}=h\left(\mathbf{I P}\left(\mathbf{t}_{n}^{-}\right)-\mathbf{Y}_{n}\right)$, where $h$ is a function dependent on the control rule, the cost of the control rule is given by $g_{2}\left(\mathbf{I P}\left(\mathbf{t}_{n}^{-}\right)-\mathbf{Y}_{n}\right)$, with $g_{2}=G_{2} \circ h$.

Before starting with the actual computation of these costs we discuss some properties related to the expected long run average cost associated with a stochastic process. The average cost associated with a positive function $l$ (or if $l$ is a function with bounded variation) and a 
stochastic process $\mathbf{X}$ is given by

$$
\lim _{t \uparrow \infty} \mathbb{E}\left(\frac{1}{t} \int_{0}^{t} l(\mathbf{X}(s)) d s\right) .
$$

Assume now that the time-stationary distribution for the stochastic process $\mathbf{X}$ exists, and $\mathbf{X}_{\infty}^{c}$ denotes a random variable distributed with this time- stationary distribution of the process $\mathbf{X}$. Using Fubini's theorem in the previous relation yields that

$$
\lim _{t \uparrow \infty} \frac{1}{t} \int_{0}^{t} l(\mathbf{X}(s)) d s=l\left(\mathbf{X}_{\infty}^{c}\right) \text { a.s. }
$$

Having obtained almost surly convergence, the next step in order to obtain $\mathrm{L}^{1}$ convergence is use Theorem 13.7 of Williams (1991), and Scheffé's Lemma (Williams (1991)) establishing a sufficient and necessary condition for $\mathrm{L}^{1}$ convergence:

$$
\begin{gathered}
\lim _{t \uparrow \infty} \mathbb{E}\left(\frac{1}{t} \int_{0}^{t} l(\mathbf{X}(s)) d s\right)=\mathbb{E}\left(l\left(\mathbf{X}_{\infty}^{c}\right)\right) \\
\text { if and only if } \\
\left\{\frac{1}{t} \int_{0}^{t} l(\mathbf{X}(s)) d s: t \geq>0\right\} \text { is uniformly integrable. }
\end{gathered}
$$

One can also define costs as event-averages, in the following way. The costs related to the jumps (type I or type II) are associated with events, hence we define an event-average cost in the following manner. The event-average cost related to the series of events $\left\{\mathbf{s}_{n}: n \in \mathbb{N} \cup\{0\}\right\}$ associated with a stochastic process $\mathbf{X}$ and a positive cost-rate function $l$ is given by

$$
\lim _{n \uparrow \infty} \mathbb{E}\left(\frac{1}{n} \sum_{j=1}^{n} l\left(\mathbf{X}\left(\mathbf{s}_{j}\right)\right)\right) .
$$

Similarly as relation (4.2) for the continuous case, we obtain that

$$
\begin{gathered}
\lim _{n \uparrow \infty} \mathbb{E}\left(\frac{1}{n} \sum_{j=1}^{n} l\left(\mathbf{X}\left(\mathbf{s}_{j}\right)\right)\right)=\mathbb{E}\left(l\left(\mathbf{X}_{\infty}^{e}\right)\right) \\
\text { if and only if } \\
\left\{\frac{1}{n} \sum_{j=1}^{n} l\left(\mathbf{X}\left(\mathbf{s}_{j}\right)\right): n \in \mathbb{N}\right\} \text { is uniformly integrable, }
\end{gathered}
$$

where $\mathbf{X}_{\infty}^{e}$ is a random variable distributed with the event stationary distribution $F_{\infty}^{e}$ defined by (2.4). Let us summarize relations (4.1) - (4.4) in the following theorem.

Theorem 4.1. Assuming that the time and event stationary distributions, $F_{\infty}^{c}$ resp. $F_{\infty}^{e}$, for the stochastic process $\mathbf{X}$ exist, then

$$
\lim _{t \uparrow \infty} \mathbb{E}\left(\frac{1}{t} \int_{0}^{t} l(\mathbf{X}(s))\right) d s=\mathbb{E}\left(l\left(\mathbf{X}_{\infty}^{c}\right)\right),
$$


if and only if $\left\{(1 / t) \int_{0}^{t} l(\mathbf{X}(s)) d s: t>0\right\}$ is uniformly integrable; and

$$
\lim _{n \uparrow \infty} \mathbb{E}\left(\frac{1}{n} \sum_{j=1}^{n} l\left(\mathbf{X}\left(\mathbf{s}_{j}\right)\right)\right)=\mathbb{E}\left(l\left(\mathbf{X}_{\infty}^{e}\right)\right),
$$

if and only if $\left\{(1 / n) \sum_{j=1}^{n} l\left(\mathbf{X}\left(\mathbf{s}_{j}\right)\right): n \in \mathbb{N}\right\}$ is uniformly integrable. Expressions (4.5) and (4.6) are the time-, respectively event-average costs related to the process $\mathbf{X}$ and the cost-rate function $l$. Moreover, if $\mathbf{N}(t) / t \longrightarrow \lambda$ a.s. as $t \longrightarrow \infty$ then

$$
\lim _{t \uparrow \infty} \mathbb{E}\left(\frac{1}{t} \sum_{j=1}^{\mathbf{N}(t)} l\left(\mathbf{X}\left(\mathbf{s}_{j}\right)\right)\right)=\lambda \mathbb{E}\left(l\left(\mathbf{X}_{\infty}^{e}\right)\right),
$$

and $\left\{(1 / t) \sum_{j=1}^{\mathbf{N}(t)} l\left(\mathbf{X}\left(\mathbf{s}_{j}\right)\right): t>0\right\}$ uniformly integrable.

Obviously, if the pointwise limiting distribution of the stochastic process $\mathbf{X}$ exists then it coincides with the distributions defined by relations (2.3) and (2.4). The right hand side of relation (4.7) is the time-average version of the cost defined on a set of events.

Assumption 4.2. For the case of the inventory systems considered, we assume for the rest of the paper that $\left\{(1 / t) \int_{0}^{t} l\left(\phi_{L} \mathbf{I N}(s)\right) d s: t>0\right\}$ is uniformly integrable and / or $\left\{(1 / n) \sum_{j=1}^{n} l\left(\phi_{L} \mathbf{I N}\left(\mathbf{s}_{j}\right)\right): n \in \mathbb{N}\right\}$ is uniformly integrable.

4.2. Average holding cost. Since in this case we are interested in long run time-average costs we aim to compute the expression

$$
\lim _{t \uparrow \infty} \frac{1}{t} \int_{0}^{t} \mathbb{E} f\left(\phi_{L} \mathbf{I N}(s)\right) d s .
$$

Relation (2.2) gives us a powerful tool to compute the average cost. By the definition of the demand process (2.1) the average cost equals

$$
\lim _{t \uparrow \infty} \frac{1}{t} \int_{0}^{t} \mathbb{E} f\left(\mathbf{I P}(s)-\sum_{k=1}^{\phi_{s} \mathbf{N}(0, L]} \mathbf{Y}_{k}\right) d s .
$$

Proposition 4.3. The average holding cost defined by relation (4.9) equals

$$
\mathbb{E}_{\mathbf{I P}}\left(\left(f * F_{D_{\infty}(0, L]}\right)\left(\mathbf{I} \mathbf{P}_{\infty}\right)\right)
$$

where $\mathbf{D}_{\infty}(0, L]:=\sum_{k=1}^{\mathbf{N}_{\infty}^{c}(0, L]} \mathbf{Y}_{k}$. 
Proof. As deduced in Theorem 3.2, $\mathbf{I P}(t)$ and $\mathbf{D}(t, t+L]$ are asymptotically independent. Since $\mathbf{I} \mathbf{P}_{\infty}, \mathbf{N}_{\infty}^{c}(0, L]$ and $\mathbf{Y}_{k}$ are pair by pair independent, the statement of the proposition follows immediately.

Observe that the expression for the demand process can be written in the form

$$
\mathbb{P}\left\{\sum_{k=1}^{\mathbf{N}_{\infty}^{c}(0, L]} \mathbf{Y}_{k} \leq x\right\}=\sum_{k=0}^{\infty} \mathbb{P}\left\{\mathbf{N}_{\infty}^{c}(0, L]=k\right\} F_{Y}^{k *}(x)
$$

and taking the Laplace- Stieltjes transform of this we obtain

$$
L S_{F_{D_{\infty}}}(\alpha)=\sum_{k=0}^{\infty} \mathbb{P}\left\{\mathbf{N}_{\infty}^{c}(0, L]=k\right\} L S_{F_{Y}}^{k}(\alpha)=P_{\mathbf{N}_{\infty}^{c}(0, \mathrm{~L}]}\left(L S_{F_{Y}}(\alpha)\right),
$$

where $P_{\mathbf{N}_{\infty}^{c}(0, \mathrm{~L}]}(\cdot)$ denotes the z-transform of $\mathbf{N}_{\infty}^{c}(0, L]$. In conclusion, if we can determine $P_{\mathbf{N}_{\infty}^{c}(0, \mathrm{~L}]}$ then with the previously mentioned Laplace transform inversion algorithm we obtain a piece-wise polynomial approximation for $f * F_{D_{\infty}(0, L]}$, say $P_{f * F_{D_{\infty}(0, L]}}$. We are now able to approximate equation (4.10) by

$$
\mathbb{E}_{\mathbf{I P}}\left(P_{f * F_{D \infty}(0, L]}\left(\mathbf{I P}_{\infty}\right)\right)
$$

obtaining a result which is almost up to machine precision.

4.3. Service measures. The long run event-average cost, as given by relation (4.3), of the (type I) jumps associated with the cost-rate function $g_{1}$ is of the form

$$
\lim _{n \uparrow \infty} \frac{1}{n} \sum_{j=1}^{n} \mathbb{E}\left(g_{1}\left(\mathbf{I N}\left(\mathbf{t}_{j}^{-}\right), \mathbf{Y}_{j}\right)\right)
$$

Furthermore, by the definition of the demand process it is obvious that $\mathbf{I N}\left(\mathbf{t}_{n}^{-}\right)$and $\mathbf{Y}_{n}$ are independent for any $n \in \mathbb{N} \cup\{0\}$, and $\mathbf{Y}_{n}, n \in \mathbb{N} \cup\{0\}$ are identically distributed. Using now relation (2.2) the above average cost expression equals

$$
\lim _{n \uparrow \infty} \frac{1}{n} \sum_{j=1}^{n} \mathbb{E} g_{1}\left(\mathbf{I P}\left(\mathbf{t}_{j}^{-}-L\right)-\sum_{k=1}^{\phi_{\mathbf{t}_{j}} \mathbf{N}(-L, 0]} \mathbf{Y}_{k}, \mathbf{Y}_{\infty}\right)
$$

where the notation $\mathbf{Y}_{\infty}$ stands for a random variable distributed as $\mathbf{Y}_{1}$. As deduced in section $3, \mathbf{I P}(t)$ has a pointwise limit in distribution, and by the assumptions the event stationary distribution for $\phi_{t} \mathbf{N}(0, L]$ exists. We obtain thus by relation (4.4) the following proposition. 
Proposition 4.4. The event-average cost of the type I jumps equals

$$
S M_{\text {event }}:=\mathbb{E} g_{1}\left(\mathbf{I P}_{\infty}-\sum_{k=1}^{\mathbf{N}_{\infty}^{e}(0, L]} \mathbf{Y}_{k}, \mathbf{Y}_{\infty}\right)=\mathbb{E}_{\mathrm{IP}, \mathrm{Y}_{\infty}}\left(g_{1}\left(\cdot, \mathbf{Y}_{\infty}\right) \star F_{\mathrm{D}^{\mathrm{e}}(0, \mathrm{~L}]}\left(\mathbf{I P}_{\infty}\right)\right),
$$

where $\mathbf{N}_{\infty}^{e}(0, L]$ is a random variable distributed with the event stationary distribution for $\mathbf{N}$, given by

$$
\lim _{n \uparrow \infty} \frac{1}{n} \sum_{j=1}^{n} \mathbb{P}\left\{\phi_{\mathbf{t}_{j}} \mathbf{N}(-L, 0]=k\right\} .
$$

Proof. The statement is a direct consequence of Theorem 3.2 and Remark 3.3.

Observe that by Theorem 4.1 the event-average cost (4.13) is easily convertible to a timeaverage cost expression. That is, if

$$
\lim _{t \uparrow \infty} \frac{\mathbf{N}(t)}{t}=\lambda \text { a.s., }
$$

then the long run time-average cost of the jumps, $S M_{\text {time }}$, is given as in relation (4.7) by

$$
S M_{\text {time }}=\lambda \cdot S M_{\text {event }}
$$

An intuitive example for the cost of the type I jumps would be the expected number of items short up to time $t$, which is one of the most frequently used service measures in the literature. In this case the function $g_{1}$ related to the jumps is given by

$$
g_{1}(X, Y):=(Y-X)^{+}-(-X)^{+},
$$

where $X$ is the level from where the jump occurs and $Y$ is the size of the jump. Obviously, $X:=\mathbf{I N}\left(\mathbf{t}_{k}^{-}\right)$and $Y:=\mathbf{Y}_{k}$. Figure 1. provides some intuition for the definition of the function $g_{1}$ in this case.

4.4. The cost of the control rule. As we discussed at the beginning of section 4, the type II jumps are related to the inventory position process. These jumps in the sample paths of the inventory position process occur due to placement of replenishment orders. This implies a suggestive name for this type of cost: the cost of the control rule. Thus, as in section 4.3 we can derive the following proposition. 


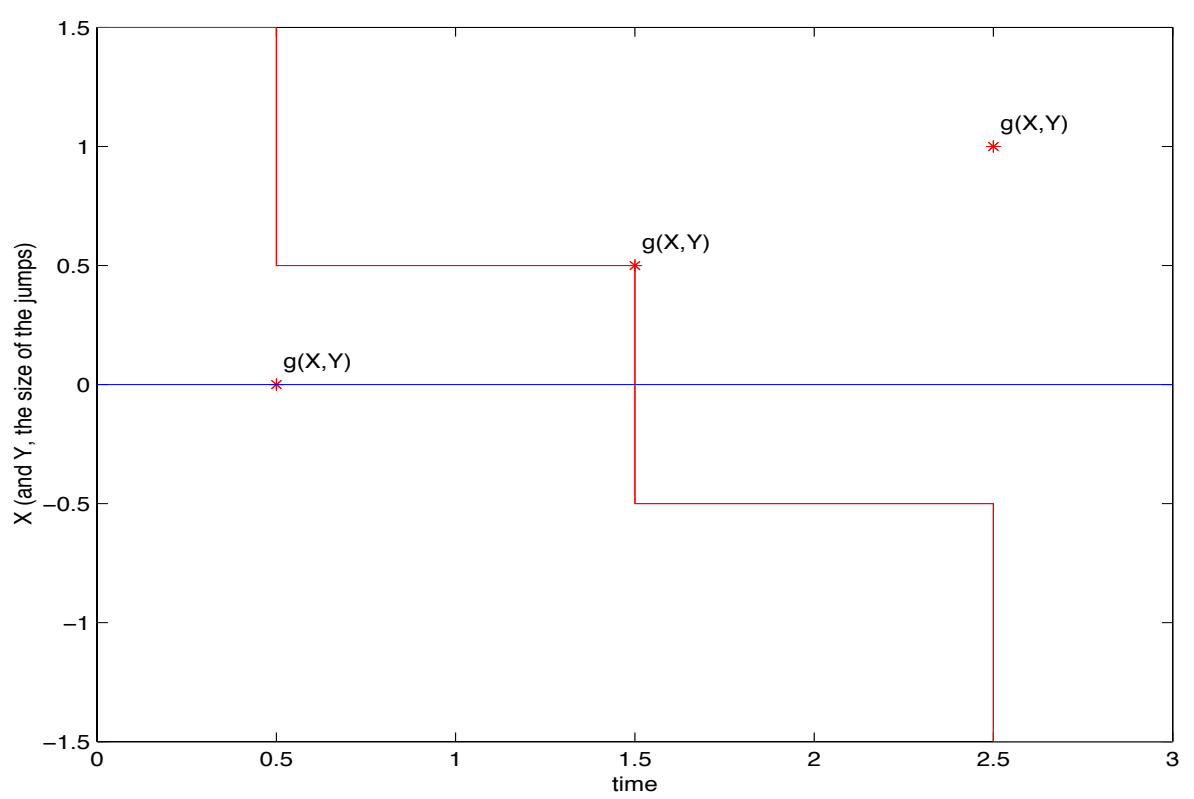

Figure 1 . The $g_{1}$ cost-rate function, related to the type I jumps

Proposition 4.5. The time-average cost of the type II jumps equals

$$
\lambda \mathbb{E} g_{2}\left(\mathbf{I P}_{\infty}-\mathbf{Y}_{\infty}\right)
$$

The most obvious example for the cost of the control rule is the setup cost. In this case the cost-rate function is given by

$$
g_{2}(A)=K 1_{\{A \leq s\}},
$$

where $K$ and $s$ are given parameters.

5. Stochastic leadtimes, Nonhomogeneous demand, And Limiting Distributions in the Cesaro SEnSE

5.1. Stochastic from nonhomogeneous with stationary versions? Let us assume for the moment that the leadtime is fixed $L>0$, and the arrival rate of the demand process is nonhomogeneous, but known, given by the function $\lambda: \mathbb{R}^{+} \longrightarrow \mathbb{R}^{+}$. As it was described 
earlier, we are interested in the limiting distribution of the leadtime demand, that is,

$$
\lim _{t \uparrow \infty} \frac{1}{t} \int_{0}^{t} \mathbb{P}\{\mathbf{D}(s, s+L] \leq x\} d s,
$$

which can be interpreted as $\phi_{\mathbf{U} t} \mathbb{P}\{\mathbf{D}(s, s+L] \leq x\}$, with $\mathbf{U}$ a uniformly distributed random variable on $[0, t]$. This, according to relation (4.5) is in fact the time stationary version of the leadtime demand, which can be further written as $\mathbb{P}\{\mathbf{D}(\mathbf{U} T, \mathbf{U} T+L] \leq x\}, T>0$. The Laplace transform of the latter is given by

$$
\exp \left(-L\left(\frac{1}{L} \int_{\mathbf{U} T-L}^{\mathbf{U} T} \lambda(v) d v\right)\left(1-L S_{F_{Y}}(\alpha)\right)\right)
$$

Hence we define a new demand rate

$$
\widehat{\lambda}:=\frac{1}{L} \int_{\mathbf{U} T-L}^{\mathbf{U} T} \lambda(v) d v
$$

the stationary version of the nonhomogeneous demand rate. Clearly, the new rate is now homogeneous but stochastic!

The message is the following. In the expression of the Laplace transform of the leadtime demand $\exp \left(-\Lambda(\mathbf{U})\left(1-L S_{F_{Y}}(\alpha)\right)\right)$, with $\Lambda(\mathbf{U})=\int_{\mathbf{U} T-L(\mathbf{U} T)}^{\mathbf{U} T} \lambda(v) d v$, it is only $\Lambda(\mathbf{U})$ which is changing or uncertain. This yields that is the distribution of $\Lambda(\mathbf{U})$ stays the same, the distribution of $L$ and $\lambda$ (or the functions $L(u)$ and $\lambda(u)$ ) can change, leading to the same stationary version of the leadtime demand, thus to the same average cost. Suppose for instance that the rate of the leadtime demand process is nonhomogeneous, with $\Lambda(\mathbf{U})=$ $\int_{\mathbf{U} T-L_{0}}^{\mathbf{U} T} \lambda(v) d v$, and fixed leadtime $L_{0}$. By keeping the distribution of $\Lambda(\mathbf{U})$ fixed, we can always transform the model into an equivalent stochastic leadtime and constant demand demand rate (say, $\left.\lambda_{0}\right)$ model. In order to achieve this, set $L(\mathbf{U} T)=\left(1 / \lambda_{0}\right) \int_{\mathbf{U} T-L_{0}}^{\mathbf{U} T} \lambda(v) d v$, yielding $\Lambda(\mathbf{U})=\lambda_{0} L(\mathbf{U} T)$, that is, stochastic leadtime, constant demand rate. The next subsection expresses the same idea with a more intuitive construction.

There is one more interesting observation to make. The limiting distribution in the Cesaro sense of the leadtime demand, $\lim _{t \uparrow \infty}(1 / t) \int_{0}^{t} \mathbb{P}\{\mathbf{D}(u, u+L] \leq x\} d u$ is almost surly equal to $\lim _{t \uparrow \infty}(1 / t) \int_{0}^{t} 1_{\{\mathbf{D}(u, u+L] \leq x\}} d u$, which is just the mathematical justification of the approximations so often used in practice. In conclusion, this means that one doesn't need to know the distribution of the whole demand process, not even that of the leadtime demand, only the fraction of time that $\mathbf{D}(L) \leq x$. 
5.2. Stochastic leadtimes vs. nonhomogeneous demand processes. Although the title of this subsection might be surprising, there is indeed an interesting relation between inventory models with stochastic leadtimes and a compound renewal demand process and inventory models with fixed leadtimes and a time-nonhomogeneous compound Poisson demand process. Consider thus a model with fixed leadtime $L>0$, and time-nonhomogeneous compound Poisson demand with rate $\Lambda(t)$. The idea is now to perform a time transformation ${ }^{2}$ $s:=\Lambda^{-1}(t)$. Intuitively, imagine that the original time axis would consist of a nonhomogeneous rubber material, which we can stretch out until the arrival moments will get into balance, such that they will correspond to a now homogeneous Poisson arrival with rate 1 on this 'new' transformed (stretched) axis. Indeed, $\Lambda(s)=\Lambda\left(\Lambda^{-1}(t)\right)=t$. While the behaviour of the demand process is cured in this way, the distances such as the leadtime $L$ are not the same as in the time before transformation: they became 'nonhomogeneous', that is, stochastic! This construction of stochastic leadtimes has a very nice property: orders do not cross in time. In this way thus we arrived to the stochastic leadtimes model described in Zipkin(1986). The long run expected average cost of the initial system with the nonhomogeneous arrivals is

$$
\lim _{t \uparrow \infty} \mathbb{E}\left(\frac{1}{t} \int_{0}^{t} f(\mathbf{I P}(u)-\mathbf{D}(u, u+L]) d u\right) .
$$

Using the change of variables $u:=\Lambda^{-1}(z)$ (the transformed time system, where the arrivals are homogeneous), and the previous relation transforms into

$$
\lim _{t \uparrow \infty} \mathbb{E}\left(\frac{\Lambda(t)}{t} \frac{1}{\Lambda(t)} \int_{0}^{\Lambda(t)} f\left(\mathbf{I P}\left(\Lambda^{-1}(z)\right)-\mathbf{D}\left(\Lambda^{-1}(z), \Lambda^{-1}(z)+L\right]\right) d \Lambda^{-1}(z)\right) .
$$

Assuming that $\Lambda(t) / t$ converges a.s. as $t$ goes to infinity to $\lambda>0$, it follows that the average cost expression becomes

$$
\lambda\left(\lim _{s \uparrow \infty} \frac{1}{s} \int_{0}^{s} f\left(\mathbf{I P}\left(\Lambda^{-1}(z)\right)-\tilde{\mathbf{D}}(z, z+\mathbf{L}]\right) \frac{1}{\lambda\left(\Lambda^{-1}(z)\right)} d z\right) .
$$

This relation, practically speaking, is the same kind of transformation as the one given in relation (4.15) between time and event averages. Besides, this relation can be interpreted as the long run average cost expression in homogeneous time, having stochastic leadtimes with probability density $1 / \lambda\left(\Lambda^{-1}(z)\right)$, less a normalization factor.

\footnotetext{
${ }^{2}$ J.B.G. Frenk, Erasmus University, Private communication
} 
The lead time $\mathbf{L}$ is now determined from the relation $\Lambda^{-1}(u)+L=\Lambda^{-1}(u+\mathbf{L}(u))(\Lambda$ can also be considered stochastic), that is, $\mathbf{L}(u)=\Lambda\left(\Lambda^{-1}(u)+L\right)-u$. With this specific $\mathbf{L}$ we can determine the ergodic stochastic process $\mathbf{U}(t)$, which drives the leadtime mechanism of Zipkin(1986), obtaining thus an equivalence between the two models. Denoting with $u$ the moment a replenishment order was placed, its arrival time $v$ is obtained in Zipkin(1986), as $v=\min \{t: t-\mathbf{U}(t) \geq u\}$ (having $t-\mathbf{U}(t)$ nondecreasing). For our model this means $v-u=\Lambda\left(\Lambda^{-1}(u)+L\right)-u$, yielding $v=\Lambda\left(\Lambda^{-1}(u)+L\right)$. Substituting this specific $v$ into the expression $v-\mathbf{U}(v)=u$, and letting $t:=\Lambda\left(\Lambda^{-1}(u)+L\right)$, yields $\mathbf{U}(t)=t-\Lambda\left(\Lambda^{-1}(t)+L\right)$. Having $\Lambda\left(\Lambda^{-1}(t)+L\right)$ increasing in $t$, $\mathbf{U}$ satisfies all the conditions of Zipkin(1986).

To conclude this section, there is an interesting observation to make.

Observation 5.1. Using stationary policies in combination with a time-nonhomogeneous demand process and fixed leadtimes can be as motivated as using stationary policies in combination with stationary demand but stochastic leadtimes. On the other hand, the demand rate can be viewed as stochastic (see the previous subsection), thus not knowing the demand rate in advance, it is natural to use a stationary policy.

\section{NuMERICAL EXAMPLES}

\subsection{Time-nonhomogeneous compound Poisson demand.}

\subsubsection{Average holding cost. In case of non-homogeneous compound Poisson demand} with arrival rate given by $\Lambda(t), t \geq 0$, we obtain that the z-transform of the time stationary distribution for the stochastic counting process is given by

$$
P_{\mathbf{N}_{\infty}^{c}(0, \mathrm{~L}]}(z)=\lim _{t \uparrow \infty} \frac{1}{t} \int_{0}^{t} \exp \left(-(1-z) \int_{s-L}^{s} \lambda(z) d z\right) d s .
$$

Therefore the average cost can again easily be computed as it was described earlier. In Figure

2. we plotted the values of the average cost of an $(s, S)$ policy with variable $s$ and $S-s$ values in case when demand is given by a non-homogeneous compound Poisson process. The demand rate function varies every (unit) interval, such that if $t \in[2 k, 2 k+1)$ then $\Lambda(t)=\lambda_{1}$ and if $t \in[2 k+1,2 k+2)$ then $\Lambda(t)=\lambda_{2}$. The individual demands follow a Gamma distribution with 
shape parameter 2.5 and scale parameter 2.5 (see Tijms(1994)). Furthermore we considered a piecewise linear cost-rate function given by

$$
f(x)= \begin{cases}-p x & \text { if } x<0 \\ h_{1} x & \text { if } 0 \leq x \leq q \\ h_{1} q+h_{2}(x-q) & \text { if } x \geq q\end{cases}
$$

where $q$ denotes a critical level of inventory, from which the inventory holding cost increases to $h_{2}$ per unit $\left(h_{2}>h_{1}>0\right)$. In the costs we also included a fixed ordering cost $K>0$ (see section 4.4) for every placement of a replenishment order. The expression (6.1) is easy to calculate, because one only needs the fraction of time that the demand has a certain rate, obtaining $1 / 2 \exp \left(-(1-z) \lambda_{1} L\right)+1 / 2 \exp \left(-(1-z) \lambda_{2} L\right)$.

6.1.2. Service measures. In case of non-homogeneous compound Poisson demand with rate $\Lambda(t)$ we obtain that the z-transform of $\mathbf{N}_{\infty}^{e}(0, L]$ is given by

$$
P_{\mathbf{N}_{\infty}(0, \mathrm{~L}]}(z)=\lim _{n \uparrow \infty} \frac{1}{n} \mathbb{E} \sum_{j=1}^{n} \exp \left(-(1-z) \int_{\mathbf{t}_{j}-L}^{\mathbf{t}_{j}} \Lambda(z) d z\right) .
$$

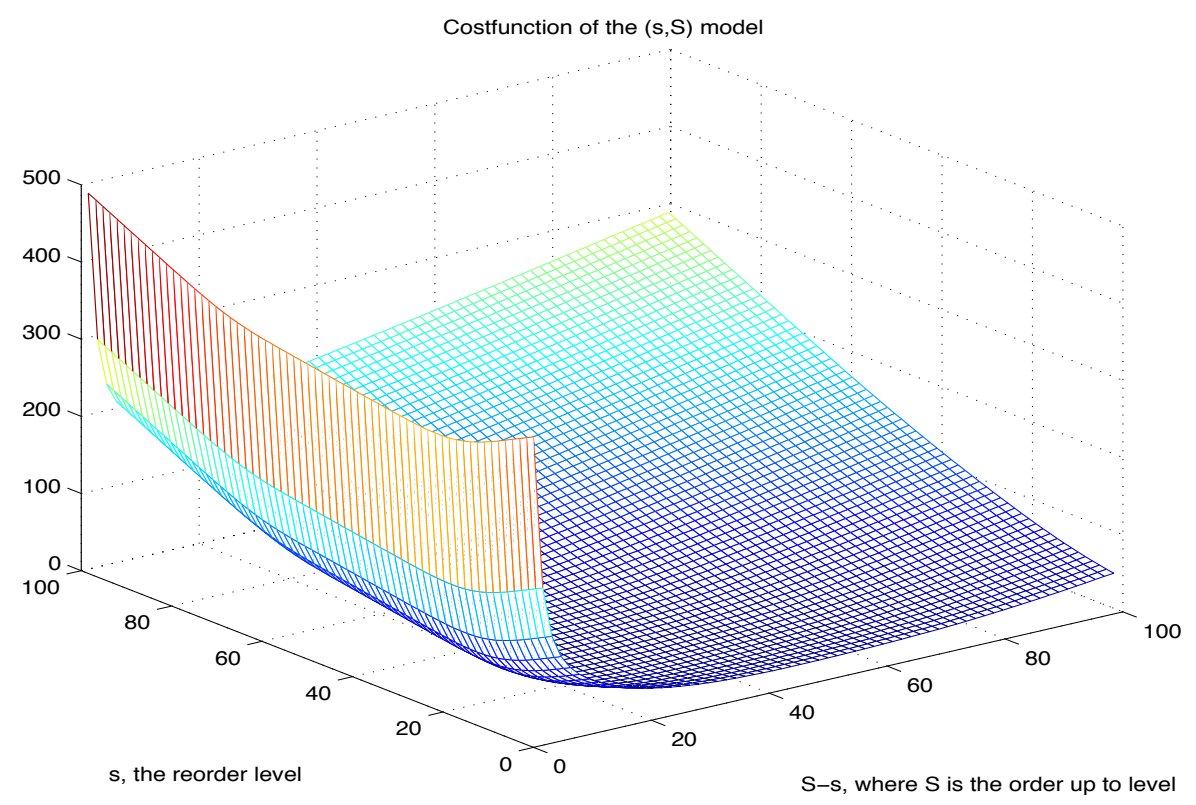

Figure 2. Average holding cost in case of an $(s, S)$ policy with nonhomogeneous compound Poisson demand; parameters are $K=20, L=$ $1, \lambda_{1}=25 / 2, \lambda_{2}=45 / 2, q=50, p=3, h_{1}=1, h_{2}=3$ ) 
Conditioning on $\mathbf{t}_{j}$ we obtain that the above equals

$$
P_{\mathbf{N}_{\infty}(0, \mathrm{~L}]}(z)=\lim _{t \uparrow \infty} \frac{1}{t} \int_{0}^{t} \exp \left(-(1-z) \int_{s-L}^{s} \Lambda(z) d z\right) \frac{\Lambda(s)}{\lambda} d s
$$

where the normalization factor $\lambda$ is given by

$$
\lambda=\lim _{t \uparrow \infty} \frac{1}{t} \int_{0}^{t} \Lambda(s) d s
$$

which is actually the rate $\lim _{t \uparrow \infty}(\mathbb{E}(\mathbf{N}(t) / t)$. This implies that relation (6.3) is in fact the time stationary transformation of the event stationary version; the transformation formula was given by relation (4.15). Both of the cases can be computed with the help of the Laplace transform inversion algorithm (see Den Iseger(2000)).

\subsection{Compound renewal demand.}

6.2.1. Average holding cost. In case of compound renewal demand, we obtain for the stochastic counting process that

$$
\lim _{t \uparrow \infty} \phi_{t} \mathbf{N}(0, L]=\lim _{t \uparrow \infty}(\mathbf{N}(t+L)-\mathbf{N}(t)) \stackrel{d}{=} \mathbf{N}_{0}(L-\mathbf{A}),
$$

where $\mathbf{A}$ is a random variable distributed with the limiting distribution of the residual life process (see Tijms(1994)) and $\mathbf{N}_{0}$ denotes the arrival process with a renewal in time point 0 .

Let us use the notation

$$
\Psi_{k}(t):=\mathbb{P}\left\{\mathbf{N}_{0}(t)=k\right\}
$$

then the probability distribution of $(6.4)$ equals $\left(\Psi_{k} \star F_{A}\right)(L)$. Straightforwardly

$$
\Psi_{k}=F_{X}^{(k-1) *}-F_{X}^{k *}, k \geq 1 \text { and } \Psi_{0}(t)=1_{\{t \geq 0\}}
$$

and the Laplace-Stieltjes transform of $F_{A}$ is given by

$$
L S_{F_{A}}(\beta)=\frac{1-L S_{F_{X}}(\beta)}{\beta \mathbb{E} X_{1}} .
$$

It follows that the two dimensional Laplace-Stieltjes transform of $\mathbf{D}(0, L]$ is given by

$$
\frac{\left(1-L S_{F_{X}}(\beta)\right)^{2} L S_{F_{Y}}(\alpha)}{\beta \mathbb{E} \mathbf{X}\left(1-L S_{F_{Y}}(\alpha) L S_{F_{X}}(\beta)\right)}+\frac{\left(1-L S_{F_{X}}(\beta)\right)}{\beta \mathbb{E} \mathbf{X}} .
$$

Thus $\beta$ is the argument of the Laplace -Stieltjes transform taken with respect to the leadtime $L$, while $\alpha$ is the argument of the Laplace -Stieltjes transform taken with respect to the individual demand $\mathbf{Y}$. With this construction we are able to calculate the long run average cost with the help of the two dimensional inversion algorithm (see Den Iseger(2000)). 


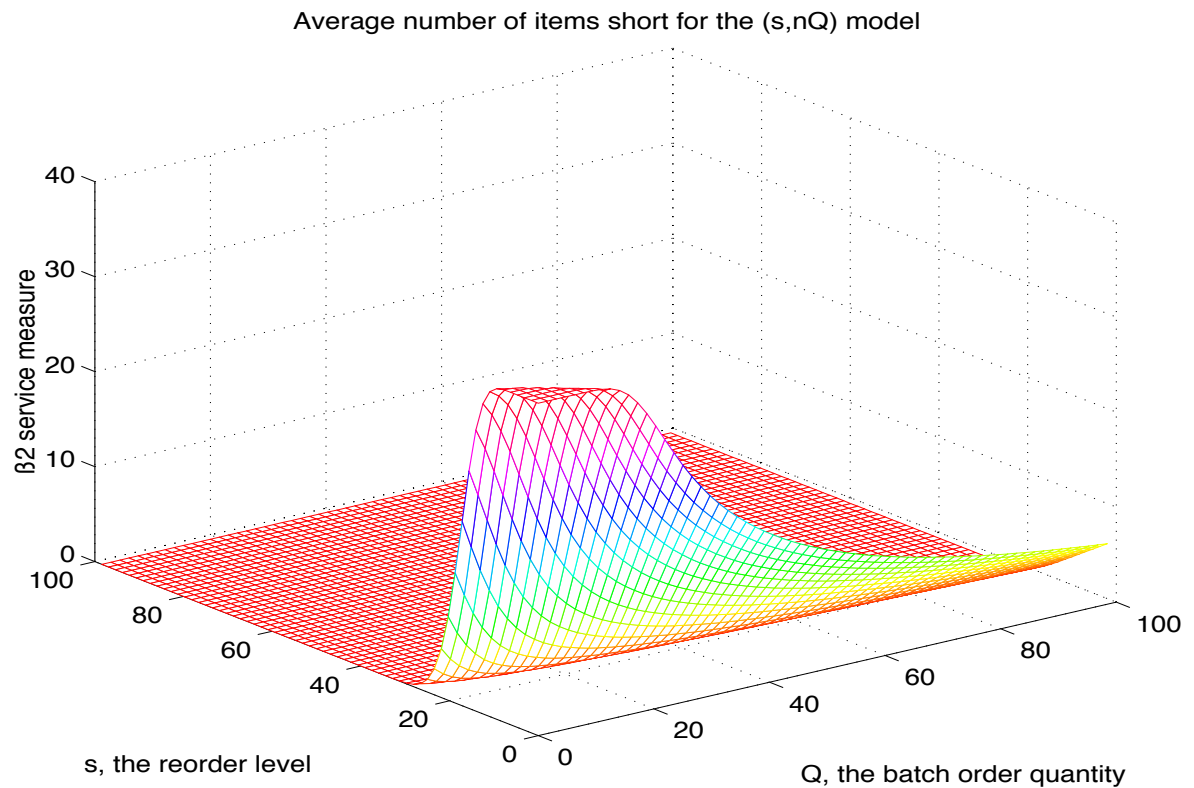

FiguRE 3. Average number $\beta_{2}$ of items short in case of an $(s, Q)$ policy $(L=0.5)$

6.2.2. Service measures. In case of compound renewal demand by a reversed time argument we obtain that

$$
\lim _{j \uparrow \infty} \mathbb{P}\left\{\mathbf{N}\left(\mathbf{t}_{j}^{-}\right)-\mathbf{N}\left(\mathbf{t}_{j}^{-}-L\right)=k\right\}=\mathbb{P}\{\mathbf{N}(L)=k\},
$$

that is $\mathbf{N}_{\infty}^{e}(0, L] \stackrel{d}{=} \mathbf{N}(L)$. Hence relation (4.12) equals

$$
\mathbb{E} g_{1}\left(\mathbf{I P}_{\infty}-\sum_{k=1}^{\mathbf{N}(L)} \mathbf{Y}_{k}, \mathbf{Y}_{\infty}\right)
$$

A special case of a general compound renewal demand process with Gamma distributed arrival process $($ shape $=5 / 2$, scale $=1 / 14$ ) and i.i.d. Gamma distributed individual demands with shape resp. scale parameters $\alpha=\beta=2.5$ are considered in case of an $(s, Q)$ control rule. The expected (time-) average number of items short, given by relation (6.6) with $g_{1}$ given by (4.16), is plotted in Figure 3., with respect to the decision variables $s$ and $Q$. 


\section{Conclusions}

The present paper extends results which are already known in the literature to a more general demand class. The generalization consists in requiring limiting distribution in Cesaro sense, thus long run averages, instead of pointwise limiting distributions. Under this assumption the model also allows nonstationary demand processes, stochastic leadtimes, all treated in a unified way. We also find an interesting equivalence for the stochastic leadtime model described in Zipkin(1986). The paper also emphasizes the importance of the asymptotical independence of the inventory position process and the leadtime demand, which in our opinion was never exploited efficiently in the literature. This is also illustrated through an important model in inventory theory. Exploiting further the asymptotical independence property, the cost structures considered are also innovative, it allows the straightforward derivation of any performance measure.

\section{Appendix A. The Proof of Theorem 3.2}

Theorem Assuming that the time- stationary distribution for the stochastic counting process $\mathbf{N}$ exists and $\mathbf{N}(t) \longrightarrow \infty$ a.s. as $t \longrightarrow \infty$, the inventory position process $\mathbf{I P}(t)$ and the leadtime demand $\mathbf{D}(t, t+L]$ are asymptotically independent. Moreover,

$$
\lim _{t \uparrow \infty} \mathbb{P}\{\mathbf{I P}(t) \leq x\}=\mathbb{P}\left\{\mathbf{I P}_{\infty} \leq x\right\}
$$

for all $x \in \mathbb{R}$ and $\mathbf{I P}_{\infty}$ is defined by relation (3.1).

Proof. Let us introduce the notations

$$
P_{n}(t):=\mathbb{P}\left\{\phi_{t} \mathbf{N}(0, L]=n\right\}
$$

$n \in \mathbb{N}$, for the time- stationary distribution of $\phi_{t} \mathbf{N}(0, L]$

$$
P_{n}(\infty):=\mathbb{P}\left\{\mathbf{N}_{\infty}^{c}(0, L]=n\right\}
$$

and the joint event

$$
\mathbf{A}_{n, n_{0}}(t):=\left(\phi_{t} \mathbf{N}(0, L]=n, \mathbf{N}(t) \geq n_{0}\right) .
$$


Proving the identity

$$
\lim _{T \uparrow \infty} \frac{1}{T} \int_{0}^{T} \mathbb{P}\left\{\mathbf{I P}(t) \leq x, \phi_{t} \mathbf{N}(0, L]=n\right\} d t=\pi P_{n}(\infty)
$$

is adequate to conclude all the statements of the theorem. The fact that IP has a pointwise limiting distribution means that for all $\varepsilon>0$, there exists an $n_{0} \in \mathbb{N}$, such that for all $n \geq n_{0}$

$$
\left|\mathbb{P}\left\{\mathbf{I P}\left(\mathbf{t}_{n}\right) \leq x\right\}-\pi(x)\right|<\varepsilon
$$

for all $x \in \mathbb{R}$. Let us now start with the expression under the limit in (A.1), that is,

$$
\mathbb{P}\left\{\mathbf{I P}(t) \leq x, \phi_{t} \mathbf{N}(0, L]=n\right\}
$$

equals the sum of probabilities

$$
\mathbb{P}\left\{\mathbf{I P}(t) \leq x, \phi_{t} \mathbf{N}(0, L]=n, \mathbf{N}(t)<n_{0}\right\}+\mathbb{P}\left\{\mathbf{I P}(t) \leq x, \phi_{t} \mathbf{N}(0, L]=n, \mathbf{N}(t) \geq n_{0}\right\} .
$$

Knowing that $\mathbf{N}(t) \longrightarrow \infty$ a.s. as $t \longrightarrow \infty$ yields

$$
\mathbb{P}\left\{\mathbf{I P}(t) \leq x, \phi_{t} \mathbf{N}(0, L]=n, \mathbf{N}(t)<n_{0}\right\} \leq \mathbb{P}\left\{\mathbf{N}(t)<n_{0}\right\} \longrightarrow 0 \text { as } t \longrightarrow \infty
$$

Relation (3.3) states that $\mathbf{I P}(t)=\mathbf{I P}\left(\mathbf{t}_{\mathbf{N}(\mathrm{t})}\right)$ for all $t \geq 0$. Suppose that $\mathbb{P}\left\{\phi_{t} \mathbf{N}(0, L]=n\right\}>0$ (otherwise the result is trivially 0 ), hence

$$
\mathbb{P}\left\{\mathbf{I P}(t) \leq x, \mathbf{A}_{n, n_{0}}(t)\right\}
$$

equals

$$
\mathbb{P}\left\{\mathbf{I P}\left(\mathbf{t}_{\mathbf{N}(\mathrm{t})}\right) \leq x \mid \mathbf{A}_{n, n_{0}}(t)\right\} \mathbb{P}\left\{\mathbf{A}_{n, n_{0}}(t)\right\} .
$$

Since in the above expression $\mathbf{N}(t) \geq n_{0}$, we know by (A.2) that

$$
\left|\mathbb{P}\left\{\mathbf{I P}\left(\mathbf{t}_{\mathbf{N}(\mathrm{t})}\right)\right\}-\pi(x)\right|<\varepsilon,
$$

independent of $\mathbf{N}(t)$ and $\phi_{t} \mathbf{N}(0, L]$. These arguments imply that

$$
\left|\mathbb{P}\left\{\mathbf{I P}\left(\mathbf{t}_{\mathbf{N}(\mathrm{t})}\right) \leq x \mid \mathbf{A}_{n, n_{0}}(t)\right\}-\pi(x)\right|<\varepsilon .
$$

The assumption that $\mathbf{N}(t) \longrightarrow \infty$ a.s. as $t \longrightarrow \infty$ also implies that

$$
\lim _{T \uparrow \infty} \frac{1}{T} \int_{0}^{T} \mathbb{P}\left\{\mathbf{A}_{n, n_{0}}(t)\right\} d t=P_{n}(\infty) .
$$

Finally we obtain that

$$
\left|\mathbb{P}\left\{\mathbf{I P}\left(\mathbf{t}_{\mathbf{N}(\mathrm{t})}\right) \leq x \mid \mathbf{A}_{n, n_{0}}(t)\right\} \mathbb{P}\left\{\mathbf{A}_{n, n_{0}}(t)\right\}-\pi(x) P_{n}(\infty)\right|
$$


is less or equal than

$$
\left|\mathbb{P}\left\{\mathbf{I P}\left(\mathbf{t}_{\mathbf{N}(\mathrm{t})}\right) \leq x \mid \mathbf{A}_{n, n_{0}}(t)\right\}-\pi(x)\right| \cdot \mathbb{P}\left\{\mathbf{A}_{n, n_{0}}(t)\right\}+\left(\mathbb{P}\left\{\mathbf{A}_{n, n_{0}}(t)\right\}-P_{n}(\infty)\right)|\pi(x)| .
$$

Taking the Cesaro limit with respect to $t$ of the above expression, and using intermediate the results (A.3) and (A.4), yields that the expression is bounded by $\varepsilon$. This completes the proof.

\section{ACKNOWLEDGEMENT}

We are indebted to Paul Zipkin for his valuable comments and suggestions, which significantly improved the paper.

\section{REFERENCES}

Axsäter, S. 1997. Simple evaluation of echelon stock $(R, Q)$ policies for two- level inventory systems. IIE Transactions 29, 661-669.

Bázsa, E., and Iseger, den P. 2001. Stochastic models with Harris chains. Tinbergen Institute discussion paper series.

Chen, F., and Zheng, Y.S. 1992. Inventory policies with quantized ordering. Naval Research Logistics 39, 285-305.

Deuermeyer, B., and Schwarz, L. 1981. A model for analysis of system level in warehouseretailer distribution systems: The identical retailer case. TIMS studies in Management Sciences 16, 163-193.

Hadley, G., and Whitin, T.M. 1963. Analysis of inventory systems. Prentice-Hall, Englewood Cliffs, NJ.

Iseger, den P.W. 2002. A new method for inverting Laplace transforms. In preperation Ross, S.M. 1970. Applied probability with optimization applications. Holden-Day, San Francisco.

Rudin, W. 1987. Real and complex analysis. Mathematics Series. McGraw-Hill, Singapore. Sahin, I. 1979. On the stationary analysis of continuous review $(s, S)$ inventory systems with constant leadtimes. Operations Research 27, 717-729. 
Sahin, I. 1990. Regenerative inventory systems: Operating characteristics and optimization. Springer-Verlag, New York.

Sigman, K. 1995. Stationary marked point processes: An intuitive approach. Stochastic Modeling. Chapman and Hall, New York.

Tijms, H.C. 1994. Stochastic models: An algorithmic approach. John Wiley \& Sons, New York.

Williams, D. 1991. Probability with martingales. Cambridge University Press, Third Reprint, Cambridge.

Zipkin, P.H. 1986. Stochastic leadtimes in continuous-time inventory models. Naval Research Logistics Quarterly 33, 763-774. 